OPEN ACCESS

Edited by:

Jacqueline Weyer,

National Institute of Communicable

Diseases (NICD), South Africa

Reviewed by:

Amy Schuh,

Centers for Disease Control and

Prevention (CDC), United States

Felicity Jane Burt,

National Health Laboratory Service

(NHLS), South Africa

*Correspondence: Olivia Wesula Lwande

olivia.lwande@umu.se

Specialty section:

This article was submitted to

Emerging and Reemerging Viruses,

a section of the journal

Frontiers in Virology

Received: 01 September 2021

Accepted: 13 January 2022

Published: 11 February 2022

Citation:

Lwande OW, Luande VN, Pereira de Freitas A, Tajedin L, Ahlm C,

Näslund J, Evander M and Bucht G

(2022) Mismatch Amplification Mutation Assays of Chikungunya Virus and O'nyong-Nyong Virus; A Simple and Reliable Method for Surveillance and Identification of Emerging Alphaviruses. Front. Virol. 2:769354.

doi: 10.3389/fviro.2022.769354

\section{Mismatch Amplification Mutation Assays of Chikungunya Virus and O'nyong-Nyong Virus; A Simple and Reliable Method for Surveillance and Identification of Emerging Alphaviruses}

\author{
Olivia Wesula Lwande ${ }^{1 *}$, Verah Nafula Luande ${ }^{1}$, Amanda Pereira de Freitas ${ }^{1}$, \\ Leila Tajedin ${ }^{2}$, Clas Ahlm ${ }^{1}$, Jonas Näslund ${ }^{3}$, Magnus Evander ${ }^{1}$ and Göran Bucht ${ }^{1}$ \\ ${ }^{1}$ Department of Clinical Microbiology, Umeå University, Umeå, Sweden, ${ }^{2}$ Department of Microsystems, University of \\ South-Eastern Norway, Vestfold, Norway, ${ }^{3}$ Swedish Defence Research Agency, CBRN, Defence and Security, Umeå, \\ Sweden
}

Background: The mosquito-borne alphaviruses chikungunya virus (CHIKV) and o'nyong-nyong virus (ONNV) are closely related Alphaviruses that belong to the Semliki forest virus serocomplex. The two viruses are associated with large outbreaks with significant morbidity. However, they are transmitted by different mosquito vectors and accordingly need different prevention strategies. The viruses are difficult to distinguish clinically and there is a lack of sensitive and specific assays that can discriminate between CHIKV and ONNV. Therefore, there is a need for new methods that may be able to determine the true burden of the diseases caused by these viruses, especially in resource-poor settings.

Method: To distinguish between CHIKV and ONNV, we designed and optimized two genetic methods, melt analysis of mismatch amplification mutation assay (Melt-MAMA) and agarose gel-based mismatch amplification mutation assay (Agarose-MAMA). The identification was based on single nucleotide polymorphisms using two competing forward primers and a common reverse primer that targeted selected sites in the envelope genes (E1 and E2). A specific shift in the melting point and mobility on agarose gels was obtained by tailing one of the two competing primers with a $\mathrm{G} / \mathrm{C}$-rich stretch of nucleotides.

Results: The melting point analyses by real-time polymerase chain reaction (qPCR Melt-MAMA) or gel-shift assay (Agarose-MAMA assay) for CHIKV and ONNV were found to be reproducible and the sensitivity of the two assays was estimated at under 100 template copies/reaction. Furthermore, no cross-reactivity with related viruses of the same serocomplex such as Mayaro virus, Ross River virus or Semliki forest virus was 
detected, or with other viruses such as Sindbis virus (Alphavirus), West Nile virus, dengue virus (Flavivirus), Inkoo virus and Tahyna virus (Orthobunyavirus). The results from the two assays were comparable when the obtained amplicons were analyzed by Melt-MAMA or by Agarose-MAMA.

Conclusion: Herein we present reliable and robust methods that can discriminate between CHIKV and ONNV. These methods can be used in well-equipped laboratories and basic clinical settings (e.g., in developing countries), as well as in field situations. The approach may also be applicable in the distinction of other closely-related mosquito-borne viruses that belong to the same serogroup.

Keywords: o'nyong-nyong, chikungunya, diagnosis, emerging, single nucleotide polymorphisms

\section{INTRODUCTION}

Chikungunya virus (CHIKV) and o'nyong-nyong virus (ONNV) are mosquito-borne alphaviruses endemic in East Africa. Aedes (Ae) albopictus and Ae. aegypti are the principal mosquito vectors for CHIKV (1), while ONNV is vectored mainly by Anopheles (An) funestus and An. gambiae, which are also malaria vectors (2).

CHIKV and ONNV are enveloped, positive-sense, singlestranded RNA viruses with a genome size of approximately $11.8 \mathrm{~kb}$ (3). They belong to the genus Alphavirus of the family Togaviridae (4-6). Their genome comprises four non-structural proteins (nsP1, nsP2, nsP3, and nsP4) and five structural proteins (C, E3, E2, 6K, and E1). The glycoproteins E1 and E2 play an important role in viral replication by facilitating membrane fusion, and the latter allows viruses to enter the cell through endocytosis $(7,8)$. The envelope proteins E1 and E2 exhibit complex and species-specific antibody reactivity, posing a challenge for diagnosis $(9,10)$. CHIKV and ONNV form a monophyletic group within the Semliki forest complex (11). The viruses are genetically distinct (12), but interestingly, it has been suggested that CHIKV mutations might have affected the evolution of ONNV and its ability to be transmitted by the Anopheles mosquitoes (13). Phylogenetic analysis shows that ONNV strains isolated from Gulu, Uganda, in 1959 and from Senegal in 1963, are closely related, while strains from Nigeria 1966, Uganda 1996, and Chad 2004 cluster in a distinct clade (14).

Since the initial discovery of CHIKV in Tanzania in 1952 (15), the virus has successively spread eastward to countries around the Indian Ocean (15). In 2004-2005, about 300,000 cases of chikungunya fever were reported on the east coast of Africa and the African islands of Lamu and Madagascar (16). Strikingly, a third of the population of Réunion Island, approximately 775,000 inhabitants, were infected during the 2005-2006 outbreaks (17). Subsequently, other countries adjacent to Réunion Island became affected as a result of the first outbreak (17).

ONNV, also discovered in Africa, has been linked with large sporadic outbreaks affecting millions of people, especially in sub-Saharan Africa. Unlike CHIKV, ONNV is confined within Africa, but ONNV morbidity in Africa is undoubtedly underestimated due to limited surveillance and under-diagnosis. However, a limited number of imported cases have acquired the virus, becoming sick after visiting East Africa on vacation
$(18,19)$. Major epidemics caused by ONNV occurred from 1959 to 1962, resulting in one of the largest arbovirus epidemics ever recorded (20). Another outbreak of ONNV in the Ivory Coast occurred in 1984-1985. It was originally called Igbo-Ora virus, but has since been found to be a lineage of ONNV. Yet another epidemic of ONNV in 1996-1997 was limited to Uganda (5).

Both of these alphaviruses are associated with severe debilitating polyarthralgia that may persist for months or years and impact patient quality of life. Other arthritogenic alphaviruses that are restrained to specific territories are Mayaro virus (MAYV) in Latin America and Ross River virus (RRV) in Australia (21). Clinically, the diseases are difficult to distinguish, because they present with common symptoms such as acute fever, skin rash, malaise, fatigue, myalgia and arthralgia, which may be severe (22).

Various serological assays, such as enzyme-linked immunosorbent assay (ELISA) and hemagglutination inhibition test (HI test), have been developed to screen for CHIKV antibodies (23). For acute infections, viral antigen detection ELISA using cross-reactive capture that identifies both CHIKV and ONNV infections has been performed (24). Past studies have demonstrated that CHIKV antibodies directed against ONNV cross-react, while ONNV antibodies exhibit a weaker cross-reaction against CHIKV $(25,26)$.

Diagnostic assays using virus isolation and molecular techniques have also been used (22). Both viruses are fastgrowing and induce cytopathic effects in mammalian and insect cell lines within 1-2 days post-infection (dpi) (23). The plaque reduction neutralization assay (PRNT) is the gold standard, but cumbersome. Unfortunately, most laboratories in regions/countries where outbreaks have been reported lack the necessary capacity to conduct virus isolation. In addition, maintaining a cold chain is challenging, interfering with virus viability. Molecular techniques such as reverse transcription (RT)-polymerase chain reaction (PCR) have been designed and used for the detection of CHIKV and ONNV RNA in clinical samples and vectors during entomological surveys. Although RT-PCR is an excellent molecular diagnostic method with high specificity and sensitivity, enabling the identification of viruses in minute samples, it requires expertise to perform the assay as well as expensive equipment and reagents (23). Likewise, 


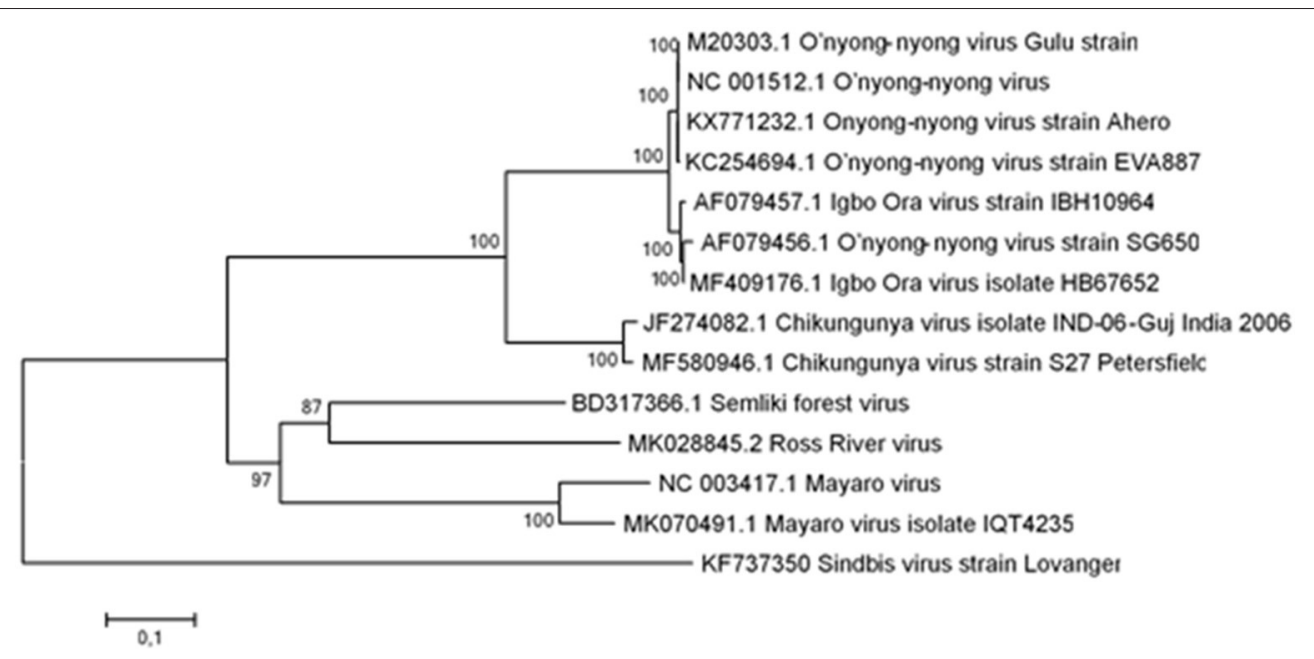

FIGURE 1 | Phylogenetic tree of the structural genes $\mathrm{E} 1$ and E2 of CHIKV, ONNV, SFV, MAYV, RRV, and SINV. The phylogenetic tree built from the structural genes (E1 and E2) with the highest log likelihood is shown. The percentage of trees in which the associated taxa clustered together is given next to the branches. The tree is drawn to scale, with branch lengths representing the number of substitutions per site.

TABLE 1 | DNA sequences of the primers used in the study and their positions.

\begin{tabular}{|c|c|c|c|}
\hline Primer & Primer position & Primer sequence $5^{\prime}$ to $3^{\prime}$ & Primer length \\
\hline ONNV $1 \mathrm{~F}$ & $176-193$ & 5'-GGGGCGGGGCGGGGCGGGGCTGAAAATCCAGGTATCTC & 38 \\
\hline CHIKV 1F w/o tail & $176-193$ & 5'-TGAAAATCCAGGTCTCCT & 18 \\
\hline ONNV $2 F$ & $595-616$ & 5'-GGGGCGGGGCGGGGCGGGGCGGAAACGTTAAGATCACAGTTG & 42 \\
\hline CHIKV 2F w/o tail & $595-616$ & 5'-GGCAACGTAAAGATCACAGTCA & 22 \\
\hline CHIKV $3 F$ w/o tail & $1,117-1,137$ & 5'-TATGAGCTGTACCCTACTATG & 21 \\
\hline ONNV/CHIKV 3R & $1,419-1,436$ & 5'-CAGCAGCATGGTAARAGT & 18 \\
\hline
\end{tabular}

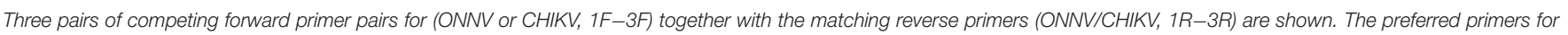

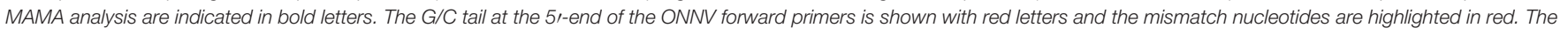
primer positions were all set according to the reference sequence of ONNV NC001512.

the advancement of high-throughput sequencing technologies such as Illumina has allowed genetic characterization through genome sequencing of CHIKV and ONNV (27). However, this is expensive and requires expertise in bioinformatics for the analysis of sequence data.

In essence, there is a dire need for a simple, cost-effective and user-friendly technique that can be applied in endemic areas. Moreover, coinfection of these viruses with other mosquitoborne viruses, for example CHIKV with dengue virus or yellow fever virus, hampers diagnosis, since most of the viruses are vectored by related mosquito species $(28,29)$.

This study aimed to evaluate and optimize mismatch amplification mutation assays (Melt-MAMA and AgaroseMAMA) to differentiate between CHIKV and ONNV. The techniques were based on single nucleotide polymorphism (SNP) analysis, melting temperature and amplicon size. The methods were found to be cost-effective and useful for the discrimination of ONNV and CHIKV. These findings may be applicable in the diagnosis and surveillance of ONNV and CHIKV.

\section{MATERIALS AND METHODS}

\section{Selection of Taxa and Design of ONNV and CHIKV Synthetic Genes}

Sequence data of the structural genes (E1 and E2) of CHIKV, ONNV, Semliki forest virus (SFV), MAYV, RRV, and Sindbis virus (SINV) were downloaded from GenBank. The analysis involved 14 nucleotide sequences. Multiple alignment of the sequences was conducted using Clustal W within the Bioedit sequence alignment editor 7.2 package (30). Evolutionary analyses were conducted in MEGA6 (31). Initial tree(s) for the heuristic search were obtained by applying the neighborjoining method to a matrix of pairwise distances estimated using the maximum composite likelihood (MCL) approach. A discrete gamma distribution was used to model evolutionary 


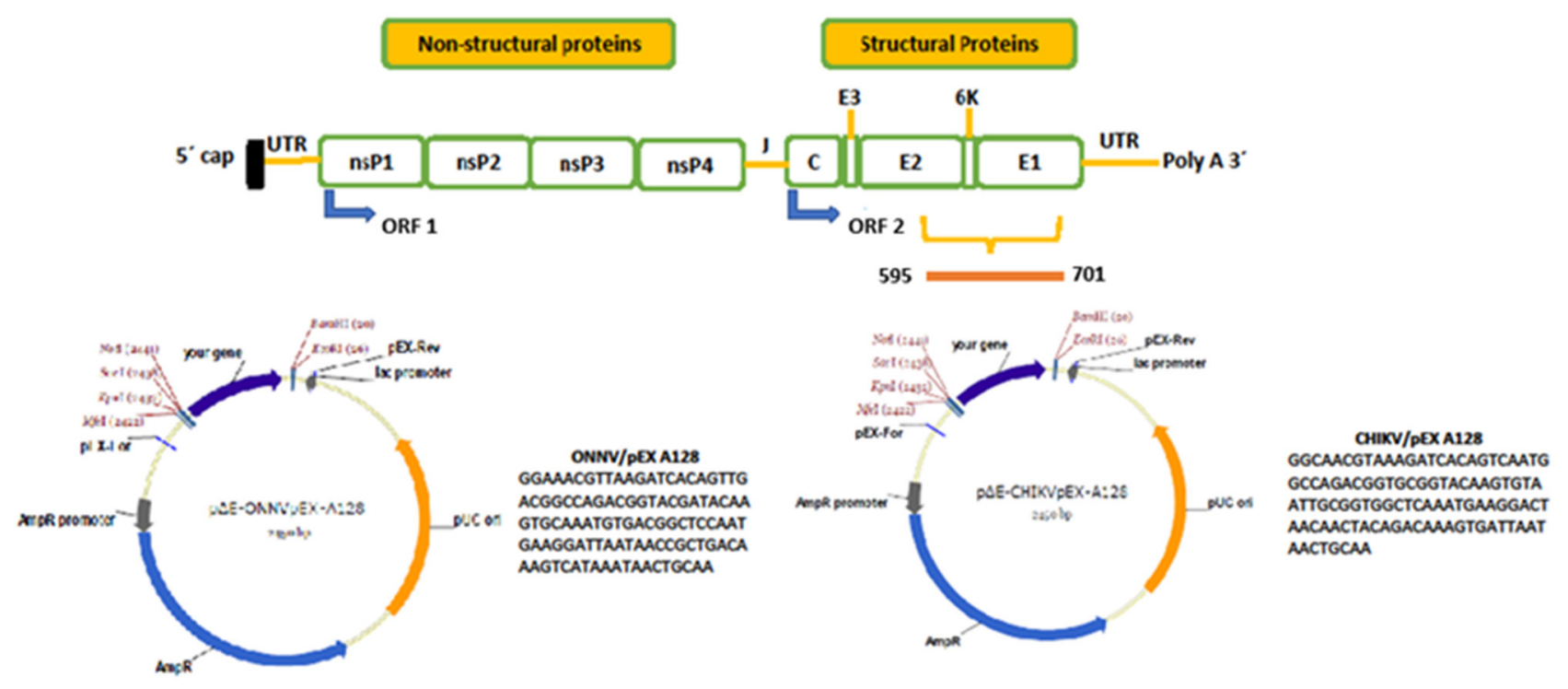

FIGURE 2 | Genetic organization of viruses of the Togaviridae family and nucleotide positions of ONNV and CHIKV synthetic genes. At the top, a schematic of the genetic organization of viruses of the Togaviridae family is shown, with non-structural and structural genes indicated. The green boxes below show the individual genes encoded by the genomes. On the right-hand side of the upper picture, the genes encoding the structural proteins are shown and the yellow staple shows the region selected for MAMA analysis. The bar below the staple shows the nucleotide positions of the synthetic genes produced, and later cloned into the corresponding plasmids ONNV/pEX A128 and CHIKV/pEX A128. Below, a simplified circular map of the two plasmids is shown, alongside the nucleotide sequences of the two synthetic genes. The plasmid maps were drawn using the SnapGene software and obtained with permission from Eurofins Genomics (Ebersberg, Germany).

rate differences among sites (two categories, $+\mathrm{G}$, parameter $=0.4499)$. All positions containing gaps and missing data were eliminated. From this data, a phylogenetic tree was created (Figure 1). There were a total of 1,891 positions in the final dataset encompassing the structural genes (E1 and E2) envelope genes. The phylogenetic tree was constructed to give an overview of the clustering of different-related alphaviruses that have been shown to cross-react in comparison to an outgroup which does not belong to the same sero-complex (Figure 1).

\section{Primer Design and Mismatch Amplification Mutation Assays}

Three pairs of competing forward primer pairs targeting the $\mathrm{E} 1$ and $\mathrm{E} 2$ genes (ONNV or CHIKV, $1 \mathrm{~F}-3 \mathrm{~F}$ ), in concert with a common reverse primer for each pair of forward primers (ONNV/CHIKV, 1R-3R), were tested against several CHIKV and ONNV strains. The specificity of the competing primers was achieved using virus specific nucleotides at the 3'-end of the forward primers (at positions -1 and -2 ), highlighted in red in Table 1. In addition, the ONNV primers were flanked at the 5/end with a G/C-rich tail containing 20 nucleotides to obtain an increase in the melting-point and gel-shift. No G/C-rich tail was added to the forward primers against CHIKV. These competing pairs of forward primers were used in combinations with the corresponding reverse primer (Table 1).

Prior to the mismatch amplification mutation assays, extraction of viral RNA from all viruses [CHIKV S27, Petersfield strain S27- (African prototype); CHIKV India 2006; ONNV
UgMP 30 (also referred as Gulu)-human, Uganda, 1959 (prototype); ONNV IbH 12628 (formerly called Igbo Ora), Nigeria; ONNV IbH 10964-human, Ivory Coast, 1984; ONNV UgH 1002-human, Uganda, 1997; ONNV DakAr 234-IP Dakar, Senegal, 1966; ONNV SG 650-human, Uganda, 1996; and IMTSSA/2004/5163 isolate from Chad 2004] was performed using the QIAmp ${ }^{\circledR}$ Viral RNA Mini Kit (QIAGEN, Hilden, Germany) according to the manufacturer's protocol (spin protocol). CHIKV strains were obtained from the Culture Collections, Public Health England, whereas the ONNV strains were taken from the Arbovirus Reference Collection (ARC) at the Division of Vector-Borne Diseases (DVBD), Centres for Disease Control and Prevention (CDC), Fort Collins, Colorado, US.

SNP genotyping was achieved by MAMA combined with either fluorescent melting curve analysis, Melt-MAMA analysis (32), or gel-shift analyses (Agarose-MAMA) visualized on 3\% agarose gels in $1 \times$ tris acetate ethylenediaminetetraacetic acid (TAE) with GelRed (Biotium Inc. Hayward, CA, US). In order to find the optimal primer combinations and PCR conditions, 19 CHIKV and seven ONNV sequences representing the two viruses were assessed for possible MAMA. From the combined observations, synthetic genes covering the corresponding E2/E1 region of the two viruses were synthesized (Figure 2) and cloned into separate pEX A128 vectors (Eurofins Genomics Ebersberg, Germany). The plasmids ONNV/pEX A128 and CHIKV/pEX A128, each containing 107 nucleotides of synthetic DNA from the E2/E1 structural gene regions of the two viruses, were quantified, diluted and aliquoted before storing at $-80^{\circ} \mathrm{C}$ until use. 

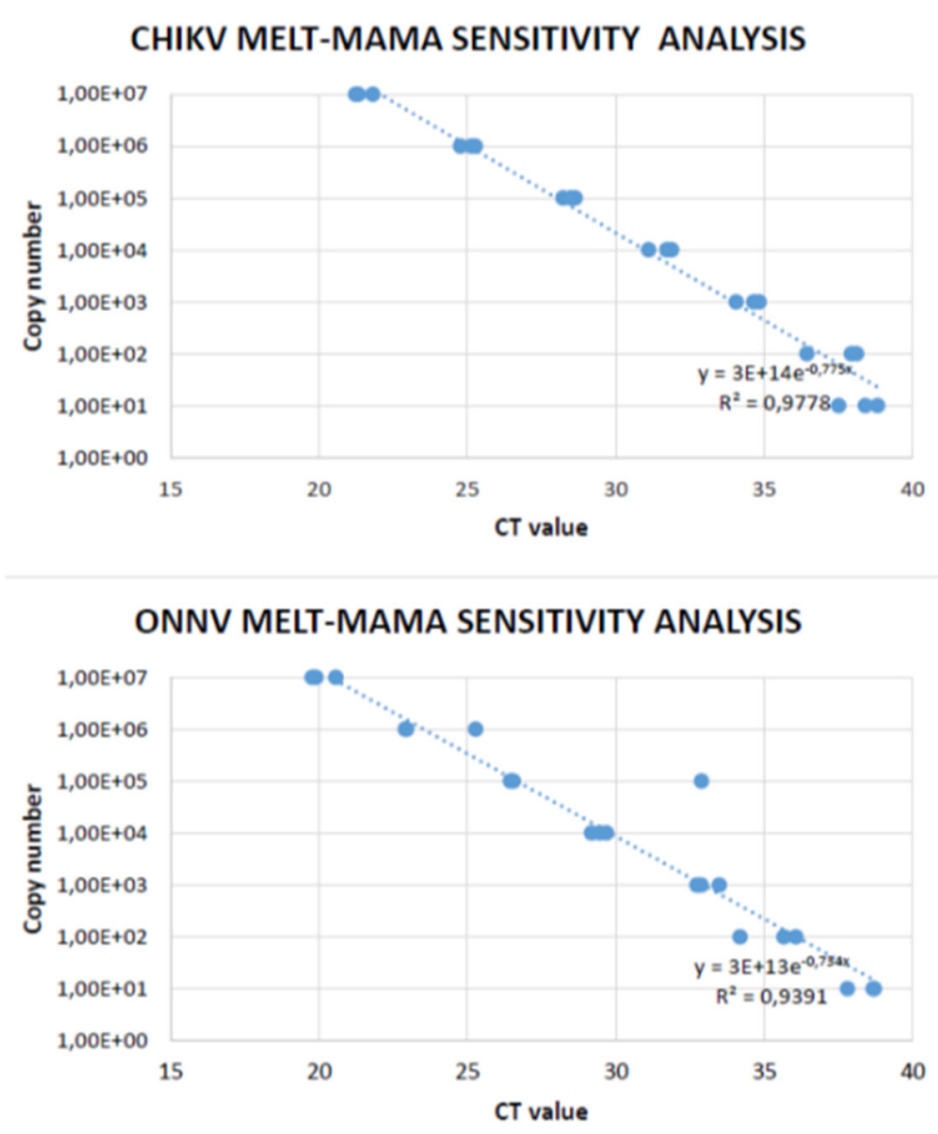

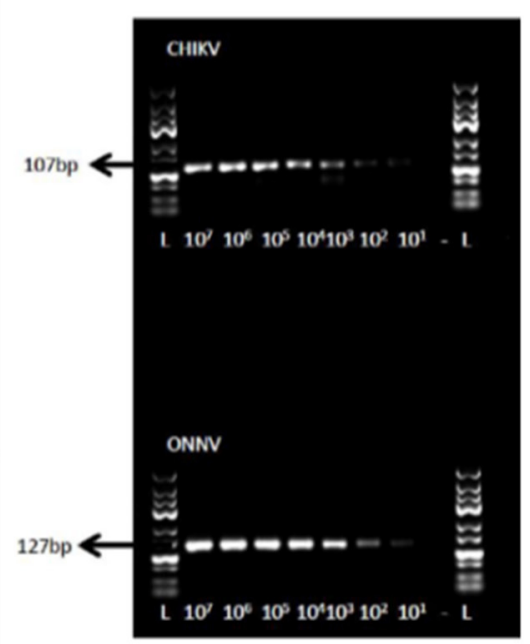

40

FIGURE 3 | CHIKV and ONNV melt-MAMA (left-hand side) and Agarose-MAMA (right-hand side) sensitivity analysis. A linear relationship between log copy number and CT value against ONNV and CHIKV plasmids using melt-MAMA and Agarose-MAMA. The sensitivity analysis by Melt-MAMA is indicated as template copies per reaction toward both viruses.

\section{PCR Conditions}

The qRT-PCR was conducted using one step KAPA SYBR ${ }^{\circledR}$ Green FAST Master Mix (Kapa Biosystems, Boston, MA, USA) and CFX96TM real-time detection system. The basic PCR conditions for Melt-MAMA were an initial $10 \mathrm{~min}$ period at $45^{\circ} \mathrm{C}$ and $2 \mathrm{~min}$ at $95^{\circ} \mathrm{C}$, followed by 40 cycles of: $5 \mathrm{~s}$ at $95^{\circ} \mathrm{C}$ and $25 \mathrm{~s}$ at $60^{\circ} \mathrm{C}$. The samples were finally heated from 60 to $95^{\circ} \mathrm{C}$ (at a rate of $0.3^{\circ} \mathrm{C}$ every $15 \mathrm{~s}$ ) before analysis. CT-values of more than 37 were considered negative.

\section{RESULTS}

\section{Optimization of the MAMA Assay}

Twenty-six virus sequences comprising $30 \mathrm{CHIKV}$ and seven ONNV were downloaded from GenBank. After alignment, potential regions within the E2/E1 genes were identified for possible MAMA assays. Three pairs of forward primers (ONNV/CHIKV) in concert with three reverse primers (ONNV/CHIKV, 1R-3R) for each region were designed and evaluated.
When dilution curves were generated for the three primer sets, the competing primers ONNV 2F/CHIKV 2F, in combination with the reverse primer (ONNV/CHIKV 2R), was preferred (see Table 1). The selection of the preferred primer set was based on the analytical sensitivity by the MAMA assays, against the plasmids carrying the two synthetic genes. The tests were evaluated from 10 -fold dilutions starting from 10 million copies per PCR reaction. A linear relationship between log copy number and CT value against both plasmids was noticed (see Figure 3)." The selected primer set was also found to be preferred when using cDNA of the virus isolates (data not shown).

The sensitivity analysis by Melt-MAMA repeatedly indicated a detection limit of less than 100 template copies per reaction for both viruses (Figure 3, left hand side). When using the AgaroseMAMA procedure, a similar sensitivity was found for agarose gel electrophoresis performed under the same conditions and gels containing 3\% agarose (Figure 3, right hand side).

To evaluate the specificity of the MAMA assays, other alphaviruses and non-related viruses were analyzed. Viruses belonging to the same Semliki forest virus complex as ONNV and CHIKV (i.e., MAYV, RRV, and SFV) were all negative in 


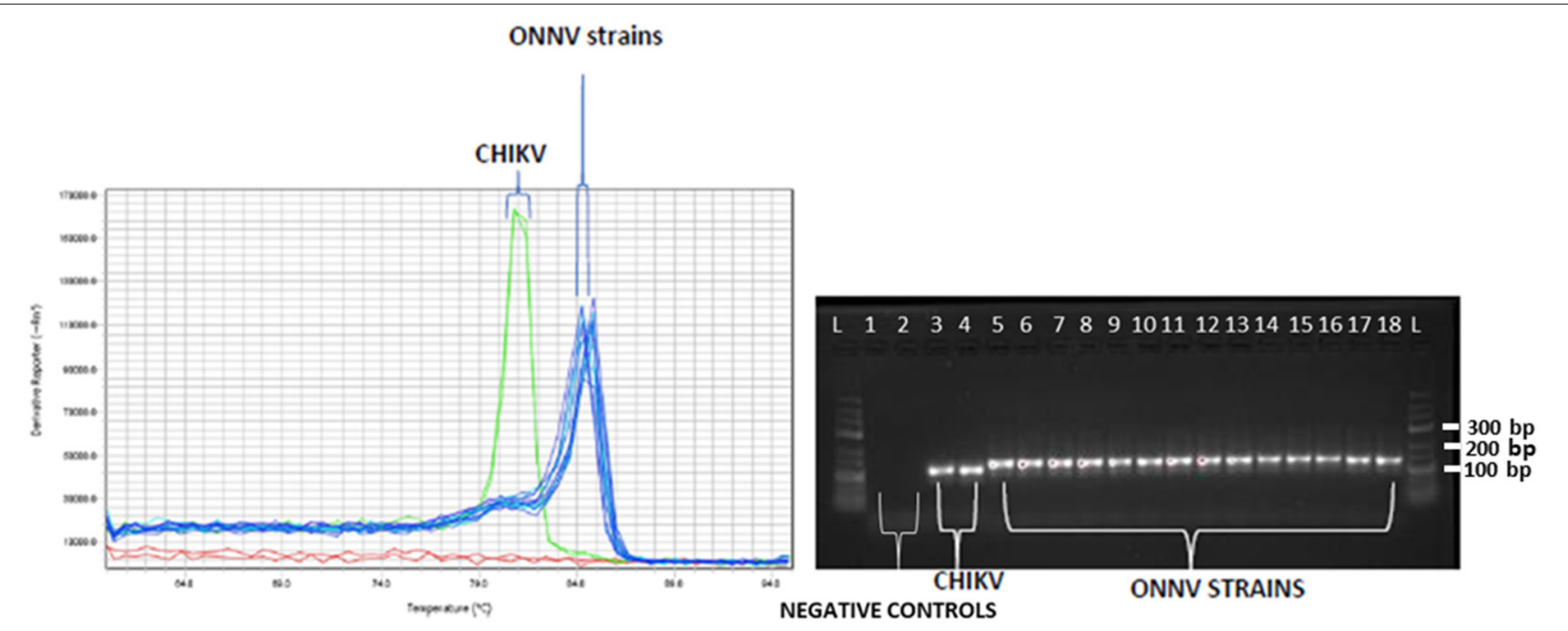

\section{Melt-MAMAs are determined by temperature-dissociation (melt) curves}

\section{Agarose-MAMAs determined by the amplicon size differences.}

FIGURE 4 | Chromatogram of Melt-MAMA and Agarose-MAMA analysis using CHIKV and ONNV strains. On the left-hand side, a chromatogram shows the results from the Melt-MAMA analysis for the two different strains of CHIKV (green peaks) and the seven ONNV isolates (blue peaks). The right-hand side shows the corresponding gel images from the results of Agarose-MAMA. From left to right, the CHIKV samples are: lane 3, CHIKV S27; and lane 4, CHIKV India 2006. The ONNV samples were run in duplicate: lane 5 and 6, UgMP 30; lane 7 and 8, lbH 12628; lane 9 and 10, IbH 10964; lane 11 and 12 , UgH 1002; lane 13 and 14, DakAr 234; lane 15 and 16, SG 650; and lane 17 and 18, IMTSSA/2004/5163.

the specificity tests. In addition, Sindbis virus, belonging to the Western Equine Encephalitis virus complex, and other less related viruses such as Flaviviruses (West Nile virus, dengue virus) and Orthobunyaviruses (Inkoo virus and Tahyna virus) were also negative. When using Agarose MAMA for the specificity testing, no bands were detected that corresponded to the correct amplicon size for ONNV and CHIKV when using the above viruses (data not shown).

\section{Evaluation of MAMA Analysis Using CHIKV and ONNV Isolates}

The results indicated when the two MAMA assays were validated with two CHIKV strains. In addition, seven ONNV strains were analyzed using the two competing forward primers (ONNV 2F/CHIKV 2F) and the common reverse primer (ONNV/CHIKV 2R).

Two major peaks were observed from the Melt-MAMA analysis (left-hand side of Figure 4). The low melting points were only noticed when using CHIKV strains and the high melting points were obtained when using the seven ONNV strains. The higher melting point peaks resulted from the G/C-rich tail attached to the ONNV primer, and the melting temperature increased from 77.2 to $84.7^{\circ} \mathrm{C}$. The insertion of 20 nucleotides into the amplicon of ONNV was not only detected due to the increase in melting-point, but also a gel-shift when using the Agarose-MAMA (right-hand side of Figure 4).

\section{DISCUSSION}

SNPs are found in the genomes of all species, and the techniques used in this study are based on SNP analysis, melting temperature and/or gel shift. The current study aimed to design, optimize and evaluate MAMA assays for the diagnosis of CHIKV and ONNV in clinical and environmental samples. Importantly, by using this approach, we believe that co-infected samples (CHIKV/ONNV) or pools of samples containing the two viruses can also be readily identified as double peaks or bands at predicted sizes by Melt-MAMA and Agarose-MAMA, respectively.

The assay was able to distinguish between ONNV and CHIKV, two closely related viruses that cross-react immunologically, posing a major challenge for diagnosis and surveillance, especially in areas where they co-circulate $(2,33)$. The sensitivity of the MAMA assays was less than 100 templates (Figure 3), and the dissociation curves from the two virus products clearly demonstrated a difference in the melting points of the two amplicons (Figure 4). Interestingly, a peak with a slightly higher melting point was noticed for the ONNV strains from Uganda (UgH 1002, 1996 and SG 650 human, 1997), but no difference in $\mathrm{G} / \mathrm{C}$ content was noticed, in comparison to the other five ONNV isolates.

The Melt-MAMA assay was able to detect all tested ONNV and CHIKV strains. The two CHIKV strains that were isolated from Ae. aegypti (old isolate) and Ae. albopictus (new isolate) mosquitoes were both detected, implying that the mutations 
acquired over a large timespan (e.g., the mutation at position 226 where alanine was substituted for valine) had no effect on detection (34).

The other variant of the assay, Agarose MAMA, demonstrated a clear difference in mobility on agarose gels between CHIKV and ONNV products.

The specificity of both MAMA assays for CHIKV and ONNV was confirmed by evaluation against related and unrelated viruses.

Compared to ordinary PCR, Melt-MAMA has added value as it is a flexible technique that can be modified for many different purposes (32). The assay is less-time consuming in that it can be used to screen for both viruses simultaneously (35). It is also less costly compared to ordinary PCR as fewer reagents are required to perform the assay, and it is possible to screen for both viruses in a single run. We also believe that the assay can detect coinfections, which is important, especially in areas where it has been difficult to pinpoint the exact cause of an outbreak due to a lack of a clear case definition criteria and lack of sensitive and specific diagnostic tests.

The Melt-MAMA assay is intended to be easy to perform, rapid and robust; it could probably be implemented in health facilities with limited resources. Besides, the assay may be useful for the demographic, epidemiological and molecular characterization of selected pathogens, with the ultimate goal of establishing a long-term surveillance system for certain arboviral diseases with the potential to cause outbreaks (35).

\section{CONCLUSION}

We conclude that the two-MAMA assays were sensitive and specific for the molecular epidemiological surveillance of ONNV and CHIKV. We believe that these findings may serve as a basis for the development and optimization of other assays for research, diagnosis and surveillance of other infectious diseases.

\section{SUMMARY}

ONNV and CHIKV are closely related viruses that are difficult to distinguish clinically as they present with similar clinical manifestations. Despite the large outbreaks linked to the viruses, there is little data available on their true burden, especially in endemic areas, most of which are resource-poor settings.

\section{REFERENCES}

1. Coffey LL, Failloux AB, Weaver SC. Chikungunya virus-vector interactions. Viruses. (2014) 6:4628-63. doi: 10.3390/v6114628

2. Rezza G, Chen R, Weaver SC. O'nyong-nyong fever: a neglected mosquito-borne viral disease. Pathog Glob Health. (2017) 111:271-5. doi: 10.1080/20477724.2017.1355431

3. Powers AM, Logue $\mathrm{CH}$. Changing patterns of chikungunya virus: re-emergence of a zoonotic arbovirus. J Gen Virol. (2007) 88:2363-77. doi: 10.1099/vir.0.82858-0

4. Rwaguma EB, Lutwama JJ, Sempala SDK, Kiwanuka N, Kamugisha J, Okware $\mathrm{S}$, et al. Emergence of epidemic O'nyong-nyong fever in southwestern
There is a lack of sensitive and specific assays that could guide accurate detection of the viruses. The current study describes the optimization and testing of a single nucleotide amplification-based assay, the mismatch amplification mutation assay, for CHIKV and ONNV, using both real-time and agarose gel techniques. The findings resulted in highly sensitive and specific assays for surveillance of ONNV and CHIKV disease that are simple and reliable. We believe that these findings can be translated to other emerging alphaviruses.

\section{DATA AVAILABILITY STATEMENT}

The original contributions presented in the study are included in the article/Supplementary Material, further inquiries can be directed to the corresponding author.

\section{AUTHOR CONTRIBUTIONS}

GB, ME, CA, OL, and JN conceived the project and sketched the draft manuscript. OL, AP, LT, and VL did the lab work. JN, $\mathrm{GB}$, and OL data analysis. All authors read and approved the final manuscript.

\section{FUNDING}

The project was supported by the Swedish Research Council grant 2017-05607 (Magnus Evander).

\section{ACKNOWLEDGMENTS}

We would like to thank the Culture Collections, Public Health, England for providing the CHIKV strains, and the Division of Vector-Borne Diseases (DVBD), Centres for Disease Control and Prevention (CDC), Fort Collins, Colorado, US, for the ONNV strains. This work was performed in part at the Umeå Centre for Microbial Research (UCMR), the Translational Research Centre (TRC), and the Arctic Research Centre at Umeå University (ARCUM).

\section{SUPPLEMENTARY MATERIAL}

The Supplementary Material for this article can be found online at: https://www.frontiersin.org/articles/10.3389/fviro. 2022.769354/full\#supplementary-material 
8. Strauss JH, Strauss EG. The alphaviruses: gene expression, replication, and evolution. Microbiol Rev. (1994) 58:491562. doi: 10.1128/mr.58.3.491-562.1994

9. Wasonga C. Serological cross-reactivity of alphaviruses to Chikungunya specific antibodies and its relationship to their structural proteins. J Biotechnol Biochem. (2017) 3:70-6. doi: 10.9790/264X-03057076

10. Fong RH, Banik SS, Mattia K, Barnes T, Tucker D, Liss N, et al. Exposure of epitope residues on the outer face of the chikungunya virus envelope trimer determines antibody neutralizing efficacy. J Virol. (2014) 88:1436479. doi: 10.1128/JVI.01943-14

11. Pezzi L, Diallo M, Rosa-Freitas MG, Vega-Rua A, Ng LFP, Boyer $\mathrm{S}$, et al. GloPID-R report on chikungunya, o'nyong-nyong and Mayaro virus, part 5: entomological aspects. Antiviral Res. (2020) 174:104670. doi: 10.1016/j.antiviral.2019.104670

12. Lanciotti RS, Ludwig ML, Rwaguma EB, Lutwama JJ, Kram TM, Karabatsos $\mathrm{N}$, et al. Emergence of epidemic O'nyong-nyong fever in Uganda after a 35year absence: genetic characterization of the virus. Virology. (1998) 252:25868. doi: 10.1006/viro.1998.9437

13. Johnson BK. O'nyong-nyong virus disease. In: The Arboviruses: Epidemiology and Ecology. Boca Raton, FL: CRC Press (2019). pp. 217-24.

14. Bessaud M, Peyrefitte CN, Pastorino BA, Gravier P, Tock F, Boete F, et al. O'nyong-nyong virus, Chad. Emerg Infect Dis. (2006) 12:1248. doi: 10.3201/eid1208.060199

15. Lumsden WH. An epidemic of virus disease in southern province, tanganyika territory, in 1952-1953 II. General description and epidemiology. Trans R Soc Trop Med Hygiene. (1955) 49:33-57. doi: 10.1016/0035-9203(55)90081-X

16. Njenga MK, Nderitu L, Ledermann JP, Ndirangu A, Logue CH, Kelly CHL, et al. Tracking epidemic chikungunya virus into the Indian Ocean from East Africa. J Gen Virolo. (2008) 89(Pt 11):2754. doi: 10.1099/vir.0.2008/005413-0

17. Renault P, Solet JL, Sissoko D, Balleydier E, Larrieu S, Filleul L, et al. A major epidemic of chikungunya virus infection on Reunion Island, France, 20052006. Am J Trop Med Hyg. (2007) 77:727-31. doi: 10.4269/ajtmh.2007.77.727

18. Tappe D, Kapaun A, Emmerich P, de Mendonca Campos R, Cadar D, Günther $\mathrm{S}$, et al. O'nyong-nyong virus infection imported to Europe from Kenya by a traveler. Emerg Infect Dis. (2014) 20:1766. doi: 10.3201/eid2010.140823

19. Bessaud M, Peyrefitte CN, Pastorino BA, Tock F, Merle O, Colpart JJ, et al. Chikungunya virus strains, Reunion Island outbreak. Emerg Infect Dis. (2006) 12:1604. doi: 10.3201/eid1210.060596

20. Williams MC, Woodall JP. O'nyong-nyong fever: an epidemic virus disease in East Africa. II. Isolation and some properties of the virus. Trans $R$ Soc Trop Med Hyg. (1961) 55:135-41. doi: 10.1016/0035-9203(61)90017-7

21. Levi LI, Vignuzzi M. Arthritogenic alphaviruses: a worldwide emerging threat?. Microorganisms. (2019) 7:133. doi: 10.3390/microorganisms7050133

22. Lwande OL, Obanda V, Bucht G, Mosomtai G, Otieno V, Ahlm C, et al. Global emergence of Alphaviruses that cause arthritis in humans. Infection Ecol Epidemiol. (2015) 5:29853. doi: 10.3402/iee.v5.29853

23. Powers AM, Roehrig JT. Alphaviruses. Methods Mol Biol. (2011) 665:17-38. doi: 10.1007/978-1-60761-817-1_2

24. Clements TL, Rossi CA, Irish AK, Kibuuka H, Eller LA, Robb ML, et al. Chikungunya and O'nyong-nyong VIRUSES in Uganda: implications for diagnostics. Open Forum Infect Dis. (2019) 6:ofz001. doi: 10.1093/ofid/ofz001

25. Blackburn NK, Besselaar TG, Gibson G. Antigenic relationship between chikungunya virus strains and o'nyong nyong virus using monoclonal antibodies. Res Virol. (1995) 146:69-73. doi: 10.1016/0923-2516(96) 80591-7
26. Chanas AC, Hubalek Z, Johnson BK, Simpson DIH. A comparative study of O'nyong nyong virus with Chikungunya virus and plaque variants. Arch Virol. (1979) 59:231-8. doi: 10.1007/BF01317418

27. Hall RA, Blitvich BJ, Johansen CA, Blacksell SD. Advances in arbovirus surveillance, detection and diagnosis. J Biomed Biotechnol. (2012) 2012:512969. doi: 10.1155/2012/512969

28. Pialoux G, Gaüzère BA, Jauréguiberry S, Strobel M. Chikungunya, an epidemic arbovirosis. Lancet Infect Dis. (2007) 7:31927. doi: 10.1016/S1473-3099(07)70107-X

29. Van den Hurk AF, Hall-Mendelin S, Pyke AT, Frentiu FD, McElroy $\mathrm{K}$, Day $\mathrm{A}$, et al. Impact of Wolbachia on infection with chikungunya and yellow fever viruses in the mosquito vector Aedes aegypti. PLoS Negl Trop Dis. (2012) 6:e1892. doi: 10.1371/journal.pntd.00 01892

30. Hall T. BioEdit: a user-friendly biological sequence alignment editor and analysis program for Windows 95/98/NT. Nucleic Acids Symp. Ser. (1999) 41:95-8. doi: 10.14601/PHYTOPATHOL_MEDITERR-14998U1.29

31. Tamura K, Stecher G, Peterson D, Filipski A, Kumar S. MEGA6: molecular evolutionary genetics analysis version 6.0. Mol Biol Evol. (2013) 30:27259. doi: 10.1093/molbev/mst197

32. Birdsell DN, Pearson T, Price EP, Hornstra HM, Nera RD, Stone N, et al. Melt analysis of mismatch amplification mutation assays (Melt-MAMA): a functional study of a cost-effective SNP genotyping assay in bacterial models. PLoS ONE. (2012) 7:e32866. doi: 10.1371/journal.pone.0032866

33. LaBeaud AD, Banda T, Brichard J, Muchiri EM, Mungai PL, Mutuku FM, et al. High rates of o'nyong nyong and Chikungunya virus transmission in coastal Kenya. PLoS Negl Trop Dis. (2015) 9:e0003436. doi: 10.1371/journal.pntd.0003436

34. Tsetsarkin KA, Vanlandingham DL, McGee CE, Higgs S. A single mutation in chikungunya virus affects vector specificity and epidemic potential. PLoS Pathog. (2007) 3:e201. doi: 10.1371/journal.ppat.0030201

35. Deekshit VK, Jazeela K, Chakraborty G, Rohit A, Chakraborty A, Karunasagar I. Mismatch amplification mutation assay-polymerase chain reaction: a method of detecting fluoroquinolone resistance mechanism in bacterial pathogens. Indian J Med Res. (2019) 149:146. doi: 10.4103/ijmr.IJMR_2091_17

Conflict of Interest: The authors declare that the research was conducted in the absence of any commercial or financial relationships that could be construed as a potential conflict of interest.

Publisher's Note: All claims expressed in this article are solely those of the authors and do not necessarily represent those of their affiliated organizations, or those of the publisher, the editors and the reviewers. Any product that may be evaluated in this article, or claim that may be made by its manufacturer, is not guaranteed or endorsed by the publisher.

Copyright (c) 2022 Lwande, Luande, Pereira de Freitas, Tajedin, Ahlm, Näslund, Evander and Bucht. This is an open-access article distributed under the terms of the Creative Commons Attribution License (CC BY). The use, distribution or reproduction in other forums is permitted, provided the original author(s) and the copyright owner(s) are credited and that the original publication in this journal is cited, in accordance with accepted academic practice. No use, distribution or reproduction is permitted which does not comply with these terms. 